\title{
Group Power Constraint Based Wi-Fi Access Point Optimization for Indoor Positioning
}

\author{
Qiaolin $\mathrm{Pu}^{1}$, Mu Zhou ${ }^{1}$, Fawen Zhang ${ }^{2}$, and Zengshan Tian ${ }^{1}$ \\ ${ }^{1}$ Chongqing Key Lab of Mobile Communications Technology, Chongqing University of Posts and \\ Telecommunications \\ Chongqing, 400065, China \\ [e-mail: puql@cqupt.edu.cn, zhoumu@cqupt.edu.cn, tianzs@cqupt.edu.cn] \\ ${ }^{2}$ Computer Science \& Technology College, Chongqing University of Posts and Telecommunications \\ Chongqing, 400065, China \\ [e-mail: zhangfw@cqupt.edu.cn] \\ *Corresponding author: Qiaolin Pu
}

Received January 13, 2017; revised July 14, 2017; accepted December 23, 2017; published May 31, 2018

\begin{abstract}
Wi-Fi Access Point (AP) optimization approaches are used in indoor positioning systems for signal coverage enhancement, as well as positioning precision improvement. Although the huge power consumption of the AP optimization forms a serious problem due to the signal coverage requirement for large-scale indoor environment, the conventional approaches treat the problem of power consumption independent from the design of indoor positioning systems. This paper proposes a new Fast Water-filling algorithm Group Power Constraint (FWA-GPC) based Wi-Fi AP optimization approach for indoor positioning in which the power consumed by the AP optimization is significantly considered. This paper has three contributions. First, it is not restricted to conventional concept of one AP for one candidate AP location, but considered spare APs once the active APs break off. Second, it utilizes the concept of water-filling model from adaptive channel power allocation to calculate the number of APs for each candidate AP location by maximizing the location fingerprint discrimination. Third, it uses a fast version, namely Fast Water-filling algorithm, to search for the optimal solution efficiently. The experimental results conducted in two typical indoor Wi-Fi environments prove that the proposed FWA-GPC performs better than the conventional AP optimization approaches.
\end{abstract}

Keywords: Wi-Fi, indoor positioning, access point optimization, power constraint, location fingerprint discrimination

This work was supported in part by the Program for Changjiang Scholars and Innovative Research Team in University (IRT1299), National Natural Science Foundation of China (61301126), and Special Fund of Chongqing Key Laboratory (CSTC). 


\section{Introduction}

In the past decade, the indoor positioning systems based on Wi-Fi network have caught significant attention [1-5]. Since the Wi-Fi Access Points (APs) are widely deployed in public hotspot areas, many kinds of positioning schemes with various signal measurements, like the Received Signal Strength (RSS), Signal-to-noise Ratio (SNR), Time-of-arrival (TOA), and Angle-of-arrival (AOA), are developed [6-12]. Among them, the RSS is more favored in indoor Wi-Fi positioning due to the free and easy access to Wi-Fi RSS, as well as cost efficiency in RSS recording [13-16].

The two main categories involved in RSS based indoor Wi-Fi positioning are the location fingerprint and trilateration based positioning schemes [17-22]. The former one estimates the target locations by matching the newly recorded RSSs against the pre-constructed radio map, while the latter one conducts the positioning based on the calculation of the distances from at least three hearable APs to the target. Both of them suffered from the positioning ambiguity as the AP number increases. To address this problem, some literatures used AP optimization approaches to improve positioning precision, which ignored the fact that the huge power consumption of the AP optimization forms a serious problem.

Power consumption in positioning system usually refers to time overhead which exists in two phases, the off-line calibration phase and on-line positioning phase. The off-line phase involves in the AP optimization and database construction and the on-line phase involves in data processing of estimating the position. This paper considers the power consumption of AP optimization in the off-line phase. AP optimization process aims at constructing a specific objective function which focuses on the demanding factors, and then searching for the optimal solution via searching algorithm which produce the most of the power consumption.

In the past decade, the Wi-Fi AP optimization has been studied extensively. The author in [23] proposed an AP filtering approach that aims at simplifying the positioning radio maps, and meanwhile improving positioning accuracy by removing the useless APs which do not contribute significantly during the positioning. These APs can be clearly identified and removed from the radio map by setting the RSS level based on threshold. SNR maximization based AP placement scheme was proposed in [24]. It regarded the location discriminant information and degree of unstable measurements as the signal and noise respectively. The principle of this scheme is to maximize the signal and minimize the noise simultaneously. In [25], the author attempted to deploy a WLAN which guaranteed the requested communication Quality of Service (QoS), as well as reduced the localization error. It introduced two indicators to evaluate network throughput and positioning accuracy, which significantly contributed to the optimization model. The author in [26] proposed an AP placement optimization approach based on the lowest positioning error bound which was derived by the theoretical relationship between positioning error and various signal distributions by using Fisher Information Matrix (FIM). In addition, the authors in [27] invented a weighted kernel function by which the impact of different APs is differentiated. It can help a lot in improving the accuracy of indoor WLAN localization. However, these approaches do not take the power consumed by the APs into account and may lead to the huge power consumption problem. The authors in [28-32] claim that the huge power consumption problem definitely exists when there are a large number of candidate APs in target environment. We clarify that the goal of this paper is not to treat the problem of power consumption independent from the design of an indoor Wi-Fi positioning system. But instead, we propose a new AP optimization approach, which not only improves the positioning accuracy, but also reduces the power consumption by choosing the 
best group of candidate AP locations.The performance of RSS based indoor Wi-Fi positioning significantly depends on the environmental and measurement properties such as physical layout. In our experiments, the proposed FWA-GPC is examined in two typical indoor Wi-Fi environments. We also compare the proposed approach with three conventional AP optimization approaches, including the Random [33], which chooses AP locations randomly, Max Mean [34], and Information Gain-based (InfoGain) [35] approaches. Max Mean approach assigns the higher importance to the candidate AP locations which make the RPs have stronger average signal strength values. InfoGain approach distributes higher confidence to more discriminative candidate AP locations, and then the candidate AP locations with the highest InfoGain is selected as the optimal AP locations. The experimental results show that the conventional approaches undergo performance degradation due to the lack of the joint consideration of location fingerprint discrimination and power consumption.

The remainder of the paper is organized as follows. In Section 2, we review the Water-filling Model from three aspects: Model construction, Conventional solution, and Fast solution. On the base of above description, Section 3 presents our AP optimization approach, which combined with water-filling model and fast solution. The extensive experiments are provided in Section 4. Finally, we conclude the paper and provide the future directions in Section 5 .

\section{Review of Water-filling Model}

Our system chooses the best group of candidate AP locations which most contributes to the location fingerprint discrimination and power consumption for indoor positioning. It is based on the concept of water-filling model, which has been widely used in adaptive channel power allocation [36-38]. The basic idea of this model is to allocate power to each channel according to the channel condition for the sake of maximizing system capacity. Here, a general introduction to water-filling model used for power allocation is given below.

\subsection{Model Construction}

It is all known that in the multi-channel wireless system with a given total power constraint at the transmitter, allocating equal power to each channel will result in the wastefulness of system resources due to the frequency-selective difference among the channels. To solve this problem, the optimal power allocation scheme is used to assist in obtaining good performance of system capacity. The analysis about the relations between the power allocation and system capacity is as follows.

The expression of system capacity, $C$, based on Shannon theory is:

$$
C=B \log _{2}\left(1+\frac{P}{N_{0}}\right)
$$

where $B$ is bandwidth; $P$ is signal power; and $N_{0}$ is noise power.

Supposing that there are two channels with signal power $P_{1}$ and $P_{2}$, where $P=P_{1}+P_{2}$, respectively, we can calculate that

$$
C=B \log _{2}\left(1+\frac{p_{1}+p_{2}}{N_{0}}\right)=B \log _{2}\left(1+\frac{p_{1}}{N_{0}}\right)+B \log \left(1+\frac{p_{2}}{p_{1}+N_{0}}\right)
$$

From (2), we can find that the first channel gives rise to the interference to the second channel since the signal power of the first channel turns into the noise power with respect to the second channel. Therefore, the power allocation scheme is widely considered as one of the 
key technologies for system capacity-achieving. In particular, the water-filling model, as one of the quintessential adaptive power allocation schemes, allocates power on the basis of SNR. The description of this model is shown in (3).

$$
\begin{aligned}
& \left(p_{1}, \cdots, p_{n}\right)=\arg \max \sum_{n \in N} B \log _{2}\left(1+S N R_{n} p_{n}\right) \\
& \text { subject to: } \sum_{n \in N} p_{n}=P_{T}^{n \in N} p_{n} \geq 0,(n=1,2, \cdots, N)
\end{aligned}
$$

where $N$ is channel number; $S N R_{n}$ is the SNR with respect to the $n$-th channel; $p_{n}(n=1,2, \cdots, N)$ is the optimal power allocated to the $n$-th channel; and $P_{T}$ is the total transmit power.

\subsection{Conventional Solution}

For the sake of generality, we modify (3) into a modified water-filling model

$$
\begin{aligned}
& \left(p_{1}^{\prime}, \cdots, p_{n}^{\prime}\right)=\arg \max \sum_{n \in N} B \log _{2}\left(1+S N R_{n} p_{n}^{\prime}\right) \\
& \text { subject to: } \quad \sum_{n \in N} p_{n}^{\prime}=P_{T}
\end{aligned}
$$

where $p_{n}^{\prime}$ is the modified power of the $n$-th channel. Using the Lagrange multiplier approach [39], we obtain

$$
L=\sum_{n=1}^{N} B \log _{2}\left(1+S N R_{n} p_{n}^{\prime}\right)-\mu\left(\sum_{n=1}^{N} p_{n}^{\prime}-P_{T}\right)
$$

where $\mu$ is Lagrange multiplier. By setting $\frac{\partial L}{\partial p_{n}^{\prime}}=0$, we have

We convert (6) into

$$
\frac{\partial L}{\partial p_{n}^{\prime}}=\frac{B}{\ln 2} \times \frac{S N R_{n}}{1+S N R_{n} p_{n}^{\prime}}-\mu=0
$$

$$
p_{n}^{\prime}=\frac{B}{\ln 2} \times \frac{1}{\mu}-\frac{1}{S N R_{n}}(n=1,2, \cdots, N)
$$

Then, the optimal solution to water-filling model is

$$
p_{n}=\left[\frac{B}{\ln 2} \times \frac{1}{\mu}-\frac{1}{S N R_{n}}\right]_{0}(n=1,2, \cdots, N)
$$

where the notation $[\bullet]_{0}=\max (\bullet, 0)$ and $\mu$ is water-filling level.

From (8), we can find that the value $p_{n}(n=1,2, \cdots, N)$ is determined by water-filling level. Therefore, the conventional solution is based on searching for the most proper water-level. As one of the most representative algorithms used for solving this problem, the iterative water-filling algorithm involves two main steps as follows.

Step 1: Initialize the parameter $k=1$, and then substitute (7) into $\sum_{n=1}^{N-k+1} p_{n}^{\prime}=P_{T}$ to obtain

$$
\mu=\frac{B}{\ln 2}\left(\frac{N-k+1}{P_{T}+\sum_{n=1}^{N-k+1} \frac{1}{S N R_{n}}}\right)
$$

The value $p_{n}^{\prime}(n=1,2, \cdots, N)$ can be calculated by substituting (9) into (7).

Step 2: Set $p_{N-k+1}^{\prime}=0$ and $k=k+1$ when the negative modified power exists, and then go back to step 1 to recalculate the values $\mu$ and $p_{n}^{\prime}(n=1,2, \cdots, N)$. This process ends until all the modified power is nonnegative, as shown in Fig. 1. 


\subsection{Fast Solution}

Without iterative searching for water-level, the procedure of fast solution [40] is discussed as follows.

From (7), we obtain

$$
\frac{B}{\ln 2} \times \frac{1}{\mu}=p_{n}^{\prime}+\frac{1}{S N R_{n}}
$$

Since the value $\mu$ is a constant, one has

$$
\frac{B}{\ln 2} \times \frac{1}{\mu}=\frac{1}{N} \sum_{n=1}^{N}\left(p_{n}^{\prime}+\frac{1}{S N R_{n}}\right)=\frac{1}{N}\left(P_{T}+\sum_{n=1}^{N} \frac{1}{S N R_{n}}\right)
$$

Substituting (9) into (11), we have

$$
p_{n}^{\prime}=\frac{1}{N}\left(P_{T}+\sum_{n=1}^{N} \frac{1}{S N R_{n}}\right)-\frac{1}{S N R_{n}}(n=1,2, \cdots, N)
$$

Then, based on (12), two cases are considered respectively to obtain the optimal solution to water-filling model.

Case 1: All the modified power $p_{n}^{\prime}(n=1,2, \cdots, N)$ is not negative.

In this case, we set $p_{n}=p_{n}^{\prime}(n=1,2, \cdots, N)$, which means the solution to water-filling model equals to the one to the modified water-filling model in (4).

Case 2: The negative modified power exists.

In this case, there are two main steps involved.

Step 1: Rank the modified power in descending order, $Q_{1} \geq \cdots \geq Q_{N}$, where $Q_{n} \geq 0(n=1,2, \cdots, k)$ and $Q_{n}<0(n=k+1, k+2, \cdots, N)$.

Step 2: Set the latter $N-k$ modified power as zero and reduce each of the former $k$ modified power by $\frac{1}{k} \sum_{n=k+1}^{N}\left(-Q_{n}\right)$ simultaneously with the purpose of preserving the total transmit power unchanged, as shown in Lemma 1. Then, save $\left\{Q_{1} \cdots Q_{N}\right\}$ into the set of modified power $\left\{p_{1}^{\prime} \cdots p_{N}^{\prime}\right\}$.

Lemma 1: For any given value $\Delta\left(0 \leq \Delta \leq P_{T} / N\right)$, we notate $\left\{p_{n}^{\prime}, n=1,2, \cdots, N\right\}$ and $\left\{\tilde{p}_{n}^{\prime}, n=1,2, \cdots, N\right\}$ as the solutions to the modified water-filling model as the total transmit power equals to $P_{T}$ and $P_{T}-N \Delta$ respectively. When $p_{n}^{\prime} \geq \Delta(n=1,2, \cdots, N)$, we set $\tilde{p}_{n}^{\prime}=p_{n}^{\prime}-\Delta(n=1,2, \cdots, N)$.

Proof: Based on (12), when the total transmit power equals to $P_{T}-N \Delta$, we can calculate that

$$
\tilde{p}_{n}^{\prime}=\frac{1}{N}\left(P_{T}-N \Delta+\sum_{n=1}^{N} \frac{1}{S N R_{n}}\right)-\frac{1}{S N R_{n}}=\frac{1}{N}\left(P_{T}+\sum_{n=1}^{N} \frac{1}{S N R_{n}}\right)-\frac{1}{S N R_{n}}-\Delta=p_{n}^{\prime}-\Delta
$$

The purpose of Lemma 1 is to illustrate that for the fast solution to the modified water-filling model, when the total transmit power is reduced by $N \Delta$, the modified power for each channel is reduced by $\Delta$. In other words, when the latter $N-k$ negative modified power becomes 0 , the total of the former $k$ modified powers should be reduced by $\sum_{n=k+1}^{N}\left(-Q_{n}\right)$ to preserve the total transmit power unchanged. Then, each of the former $k$ modified power will 
be reduced by the same amount of $\frac{1}{k} \sum_{n=k+1}^{N}\left(-Q_{n}\right)$.

The two main steps mentioned before are named as cross-zero adjustment. It requires the iterative process until all the modified powers becomes nonnegative, as shown in Fig. 2.

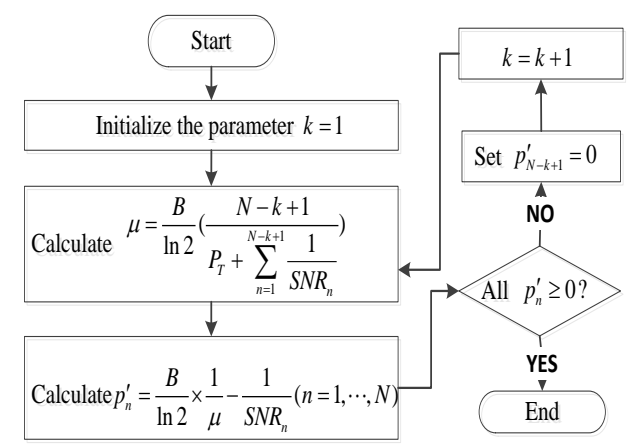

Fig. 1. Flow chart of iterative water-filling algorithm

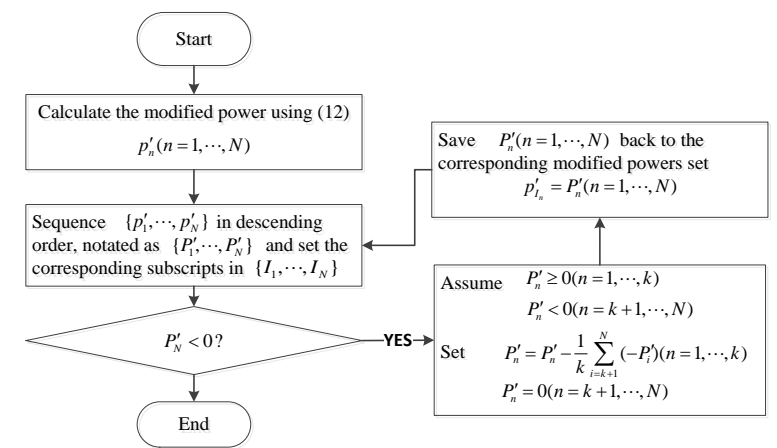

Fig. 2. Flow chart of cross-zero adjustment

\section{Approaches Description}

The conventional Wi-Fi AP optimization approaches for indoor positioning do not take the power consumed by the APs into account, and it is generally based on a natural consideration to choose as many as possible redundant APs to reduce the positioning ambiguity. However, these approaches may suffer from the huge power consumption when the number of APs chosen for positioning is significantly large. The concept of water-filling model from the adaptive channel power allocation inspires us to develop a new AP optimization approach which can improve the positioning accuracy, as well as reduce the power consumption.

\subsection{Proposed Approach}

As discussed earlier, the basic idea of the water-filling model is to allocate power to each channel according to the channel condition for the sake of maximizing system capacity. Similarly, we borrowed this concept from it to allocate APs to each candidate AP location for the sake of maximizing location fingerprint discrimination. This paper views the total transmit power as the total number of APs, channels as the candidate AP locations, Signal Noise Ratio (SNR) of each channel which represents the contribution to system capacity as the contribution of each candidate AP location which is designed to maximize location fingerprint discrimination, power allocated to each channel as the number of APs allocated to each candidate AP location, and system capacity as the location fingerprint discrimination.

Calculating importance of each candidate AP location and then ranking them is an intuitive approach for AP optimization. It treats the problem of power consumption independent from the design of indoor positioning systems, which may not favorable enough. The proposed approach focuses on measuring the location fingerprint discrimination of each group of candidate APs with a given total power, rather than assuming the equal power consumed by each AP, and then selects the group with the maximum location fingerprint discrimination.

Unlike the conventional location fingerprint discrimination approaches, which calculate the sum of Euclidean distances among all the Reference Points (RPs), a novel calculation approach is proposed in this paper. It calculates the ratio of Euclidean distances within a certain range. In practice, some APs that cannot be detected, would be given a minimum 
threshold, which will make significant contribution in the sum of Euclidean distances among all the RPs, but result in poor localization performance. In addition, if the Euclidean distances among the adjacent RPs are large, the localization errors could increase remarkably, such as the representative K-nearest Neighbor (KNN) algorithm. Based on this, our approach aims to minimize the sum of Euclidean distances among the RPs inside adjacency circle, as well as maximize the sum of Euclidean distances among the RPs outside adjacency circle. The ratio of the sum of Euclidean distances of RSS measurements outside and inside the adjacency circle with a proper radius when the AP is deployed at the $n$-th candidate AP location is defined as follows.

$$
\begin{aligned}
\gamma_{n} & =\frac{\sum_{i=1}^{M-1} \sum_{j \in D_{\text {out }}}\left[P_{i}(n)-P_{j}(n)\right]^{2}}{\sum_{i=1}^{M-1} \sum_{j \in D_{\text {in }}}\left[P_{i}(n)-P_{j}(n)\right]^{2}}(n=1,2, \cdots, N) \\
D_{\text {in }} & =\left\{j \mid \sqrt{\left(x_{i}-x_{j}\right)^{2}+\left(y_{i}-y_{j}\right)^{2}} \leq d\right\} ; D_{\text {out }}=\left\{j \mid d<\sqrt{\left(x_{i}-x_{j}\right)^{2}+\left(y_{i}-y_{j}\right)^{2}} \leq \beta d\right\}
\end{aligned}
$$

where $M$ and $N$ stand for the number of RPs and candidate AP locations; $D_{\text {in }}$ and $D_{\text {out }}$ stand for the sets of RPs inside and outside adjacency circle with respect to the circle center RP $i$; $d$ is the radius of adjacency circle; $\beta$ is a coefficient; $P_{i}(n)$ is the RSS from the $n$-th candidate AP location at RP $i$; and $\left(x_{i}, y_{i}\right)$ is the 2-D coordinate of RP $i$.

Maximizing the sum of $\gamma_{n}(n=1,2, \cdots, N)$ in all the candidate AP locations, we have

$$
f=\max \sum_{n=1}^{N} w_{n} \gamma_{n}(n=1,2, \cdots, N)
$$

where $w_{n}$ is the number of APs allocated at the $n$-th candidate AP location.

Although we borrowed the concepts of the adaptive channel power allocation to propose AP optimization, the distinction also exists. Different from the continuity property of power allocation, the discreteness property of AP optimization as well as upper-bound number of APs at each candidate AP location should be considered in our system. The reason of probably allocating more than one APs at one candidate AP location is that some important areas need to be covered by multiple APs in case of AP failure.

Based on (3) and (15), we construct the water-filling model for the AP optimization based indoor positioning as

$$
\begin{aligned}
& \left\{w_{1}, \cdots, w_{n}\right\}=\arg \max \left\{\sum_{n=1}^{N} \log _{2}\left(1+\gamma_{n} w_{n}\right)\right\}(n=1,2, \cdots, N) \\
& \text { subject to: } \sum_{n=1}^{N} w_{n}=W_{T} ; w_{n} \in\left\{\mathbb{Z}^{+}, 0\right\} ; w_{n} \leq w_{\max }^{n}
\end{aligned}
$$

where $W_{T}$ is the total number of APs also named as total power in the concept of adaptive channel power allocation and $w_{\max }^{n}$ is the upper-bound number of APs allocated at the $n$-th candidate AP location.

\subsection{Fast Version}

Considering the computation efficiency, we propose a fast version of GPC, namely FWA-GPC, to find the solution to the problem of AP optimization efficiently. It is improved from the above fast water-filling solution, whose main idea is to start with the iterative 
cross-zero adjustment, upper-bound checking, and discrete adjustment until all the values $w_{n}(n=1, \cdots, N)$ meet the constraints. The process of the proposed FWA-GPC is described as follows.

First of all, we modify (16) into

$$
\begin{aligned}
& \left\{w_{1}^{\prime}, \cdots, w_{n}^{\prime}\right\}=\arg \max \left\{\sum_{n=1}^{N} \log _{2}\left(1+\gamma_{n} w_{n}^{\prime}\right)\right\} \\
& \text { subject to: } \sum_{n=1}^{N} w_{n}^{\prime}=W_{T}
\end{aligned}
$$

where $w_{n}^{\prime}$ is the modified number of APs (hereafter referred to modified number) allocated at the $n$-th candidate AP location without considering the discreteness property and upper-bound of AP number at every candidate AP location.

Using the Lagrange multiplier approach, we have

$$
w_{n}^{\prime}=\frac{1}{\ln 2} \times \frac{1}{\mu}-\frac{1}{\gamma_{n}}(n=1,2, \cdots, N)
$$

Based on (10) and (11), one obtains

$$
w_{n}^{\prime}=\frac{1}{N}\left(W_{T}+\sum_{n=1}^{N} \frac{1}{\gamma_{n}}\right)-\frac{1}{\gamma_{n}}(n=1,2, \cdots, N)
$$

To meet the constraints in (16), the process of iterative cross-zero adjustment, upper-bound checking, and discrete adjustment is needed. As mentioned above, after calculating the value $w_{n}^{\prime}$ by (19), two cases should be considered respectively during the cross-zero adjustment.

Case 1: All the modified number $w_{n}^{\prime}(n=1,2, \cdots, N)$ is not negative.

In this case, we continue to the process of upper-bound checking.

Case 2: The negative modified number exists.

In this case, two main steps are involved.

Step 1: Rank the modified number in descending order and save the corresponding subscripts into set $\left\{I_{1}, \cdots, I_{N}\right\}$. For simplicity, the modified number is notated as $W_{1}^{\prime} \geq \cdots \geq W_{N}^{\prime}$, where $W_{n}^{\prime} \geq 0(n=1,2, \cdots, k)$ and $W_{n}^{\prime}<0(n=k+1, k+2, \cdots, N)$.

Step 2: Set the latter $N-k$ modified number $W_{n}^{\prime}(n=k+1, \cdots, N)$ as zero and reduce each of the former $k$ modified number $W_{n}^{\prime}(n=1,2, \cdots, k)$ by $\frac{1}{k} \sum_{n=k+1}^{N}\left(-W_{n}^{\prime}\right)$ simultaneously with the purpose of preserving the total AP number unchanged. Then, save $\left\{W_{1}^{\prime}, \cdots, W_{N}^{\prime}\right\}$ into the set of modified number $\left\{w_{1}^{\prime}, \cdots, w_{N}^{\prime}\right\}$. The pseudo-code of the process of cross-zero adjustment is shown in Table 1.

We conduct the iterative cross-zero adjustment until all the modified number becomes nonnegative. After the process of cross-zero adjustment, the upper-bound checking is needed for the upper-bound constraint of AP number at every candidate AP location. At this point, there are two cases to be considered respectively as follows.

Case 1: All the modified number $w_{n}^{\prime}(n=1,2, \cdots, N)$ is not larger than the upper-bound value $w_{\max }^{n}$.

In this case, we continue to the process of discrete adjustment.

Case 2: There is one or more modified number $w_{n}^{\prime}(n=1,2, \cdots, N)$ larger than $w_{\max }^{n}$. 
In this case, two main steps are involved.

Step 1: Find out the modified number which is larger than the upper-bound value and save the corresponding subscripts and values into sets $\left\{J_{1}, \cdots, J_{t}\right\}$ and $\left\{W_{1}^{\prime \prime}, \cdots, W_{t}^{\prime \prime}\right\}$ respectively.

Step 2: Modify $w_{J_{n}}^{\prime}(n=1,2, \cdots, t)$ into $w_{\max }^{J_{n}}$ and increase each of the other $N-t$ modified number by $\frac{1}{N-t} \sum_{n=1}^{t}\left(w_{\max }^{n}-W_{n}^{\prime \prime}\right)$ simultaneously with the purpose of preserving the total number of APs unchanged. The two steps above are named as the process of upper-bound checking, and the corresponding pseudo-code is shown in Table $\mathbf{1}$.

We conduct the iterative upper-bound checking until all the modified number is not larger than the upper-bound value. After the process of upper-bound checking, we convert the modified number into the discrete form. Then, two cases will be considered respectively.

Case 1: All the modified number is smaller than one.

In this case, we set $w_{n}^{\prime}=1\left(n=1,2, \cdots, W_{T}\right)$ and $w_{n}^{\prime}=0\left(n=W_{T}+1, W_{T}+2, \cdots, N\right)$.

Case 2: There is one or more modified number not smaller than one.

In this case, two main steps are involved.

Step 1: Rank the modified number in descending order and save the corresponding subscripts into set $\left\{G_{1}, \cdots, G_{N}\right\}$. Without losing generality, we notate the modified number as $W_{1}^{\prime \prime} \geq \cdots \geq W_{N}^{\prime \prime \prime}$, where $1 \leq W_{n}^{\prime \prime \prime} \leq w_{\max }(n=1,2, \cdots, r)$ and $W_{n}^{\prime \prime \prime}<1(n=r+1, r+2, \cdots, N)$. Then, we conduct the rounding down operation, notated as “ $\lfloor\cdot\rfloor$ ”, on $W_{n}^{\prime \prime}(n=1,2, \cdots, r)$ to obtain

$$
W_{n}^{\prime \prime \prime}=\left\lfloor W_{n}^{\prime \prime \prime}\right\rfloor(n=1, \cdots, r)
$$

Step 2: Increase the value $W_{n}^{\prime \prime \prime}(n=r+1, r+2, \cdots, N)$ by $\frac{1}{N-r} \sum_{n=1}^{r}\left(W_{n}^{\prime \prime \prime}-\left\lfloor W_{n}^{\prime \prime \prime}\right\rfloor\right)$ to preserve the total number of APs unchanged, and then save $\left\{W_{1}^{\prime \prime \prime} \cdots, W_{N}^{\prime \prime \prime}\right\}$ into set $\left\{w_{1}^{\prime}, \cdots, w_{N}^{\prime}\right\}$. The two steps mentioned above is named as the process of discrete adjustment, and the corresponding pseudo-code is shown in Table 1.

Table 1. Pseudo-code of three processes

\begin{tabular}{|c|c|c|}
\hline \multicolumn{3}{|c|}{ Upper-bound checking } \\
\hline 1:Begin & 1: Begin & 1: Begin \\
\hline 2: Calculate $w_{n}^{\prime}(n=1,2, \cdots, N)$ & 2: Set $S \leftarrow 0 ; t \leftarrow 0$ & 2:Rank $\quad\left\{w_{1}^{\prime}, \cdots, w_{N}^{\prime}\right\} \quad$ in \\
\hline by using (19) & 3: for $(n=1 ; n \leq N ; n++)$ & descending order, notated as \\
\hline 3:Ran $\left\{w_{1}^{\prime}, \cdots, w_{N}^{\prime}\right\}$ in descending & & $\left\{W_{1}^{\prime \prime \prime}, \cdots, W_{N}^{\prime \prime \prime}\right\}$, and save the \\
\hline order, notated as & 5: if $w_{n}^{\prime}>w_{\max }^{n}$ then & corresponding subscripts into \\
\hline$\left\{W_{1}^{\prime} \cdots W_{N}^{\prime}\right\}$, and save the & 6: \{ & set $\left\{G_{1}, \cdots, G_{N}\right\}$ \\
\hline corresponding subscripts & 7: $\quad S \leftarrow w_{\max }^{n}-w_{n}^{\prime}+S$ & 3: if $W_{1}^{\prime \prime \prime} \geq 1$ then \\
\hline into set $\left\{I_{1}, \cdots, I_{N}\right\}$ & 8: $\quad w_{n}^{\prime} \leftarrow w_{\max }^{n} ; t++$ & 4: \{ \\
\hline 4: if $W_{N}^{\prime}<0$ then & $9: \quad /\}$ & 5: $\quad$ Set $S \leftarrow 0$ \\
\hline 5: \{ & $10: /\}$ & $6:$ for $\left(n=1 ; W_{n}^{\prime \prime \prime} \geq 1, n \leq N ; n++\right)$ \\
\hline 6: $\quad$ Set $S \leftarrow 0$ & 11: $\Phi \leftarrow S / N$ & \{ \\
\hline $7: \quad$ for $\left(n=N ; W_{n}^{\prime}<0 ; n--\right)$ & 12: for $(n=1: n \leq N$ & 8: $\quad \hat{W}_{n}^{\prime \prime \prime} \leftarrow$ Round down $W_{n}^{\prime \prime \prime}$ \\
\hline 8: & $13:\{$ & $W_{n}^{\prime \prime \prime}-\hat{W}_{n}^{\prime \prime \prime}+S \rightarrow S$ \\
\hline$S \leftarrow-W_{n}^{\prime}+S$ & 14: if $w_{n}^{\prime}<w_{\max }^{n}$ then & 10: \\
\hline $\begin{array}{ll}\text { 10: } & W_{n}^{\prime} \leftarrow 0 \\
\text { 11: } & /\}\end{array}$ & 15: \{ & 11: $\quad S / N-n \rightarrow \Phi$ \\
\hline
\end{tabular}




\begin{tabular}{|c|c|c|}
\hline $\begin{array}{l}\text { 12: } \quad \Phi \leftarrow S / n \\
\text { 13: for }(k=1 ; k \leq n ; k++) \\
\text { 14: } \quad\{ \\
\text { 15: } \quad W_{k}^{\prime}-\Phi \rightarrow W_{k}^{\prime} \\
\text { 16: } \quad /\} \\
\text { 17: /\} } \\
\text { 18: for }(n=1 ; n \leq N ; n++) \\
\text { 19: }\{ \\
\text { 20: } w_{I_{n}}^{\prime} \leftarrow W_{n}^{\prime} \\
\text { 21: / } \\
\text { 22: End }\end{array}$ & $\begin{array}{l}\text { 16: } \quad w_{n}^{\prime} \leftarrow w_{n}^{\prime}+\Phi \\
\text { 17: /\} } \\
\text { 18: /\} } \\
\text { 19: End }\end{array}$ & $\begin{array}{l}\text { 12: for }(r=n ; r \leq N ; r++) \\
\text { 13: } \quad\{ \\
\text { 14: } \quad W_{r}^{\prime \prime \prime}+\Phi \rightarrow W_{r}^{\prime \prime \prime} \\
\text { 15: /\} } \\
\text { 16: else } \\
\text { 17: for }\left(n=1 ; n \leq W_{T} ; n++\right) \\
\text { 18: } \quad\{ \\
\text { 19: } \quad 1 \rightarrow W_{n}^{\prime \prime \prime} \\
\text { 20: } \quad /\} \\
21: \text { for }(r=n ; r \leq N ; r++) \\
22: \quad\{ \\
\text { 23: } \quad 0 \rightarrow W_{r}^{\prime \prime \prime} \\
\text { 24: } \quad /\} \\
\text { 25: / } \\
\text { 26: for }(n=1 ; n \leq N ; n++) \\
\text { 27: }\{ \\
\text { 28: } w_{G_{n}}^{\prime} \leftarrow W_{n}^{\prime \prime \prime} \\
\text { 29: /\} } \\
\text { 30: End }\end{array}$ \\
\hline
\end{tabular}

We conduct the iterative discrete adjustment until all the modified number becomes discrete. Finally, the AP optimization for indoor positioning is obtained based on the optimal solution to our proposed water-filling model. The flowchart of the proposed FWA-GPC algorithm is shown in Fig. 3.

Note that our approach is able to perform more efficient when the upper-bound value is set to be one at the expense of possible degradation of uncertainty of the locations for the spare APs. In such scenario, after the process of the iterative cross-zero adjustment, we do not need to conduct upper-bound checking, but continue to discrete adjustment. Understandably, there is a modification of the process of discrete adjustment as follows.

Case 1: All the modified number is smaller than one.

In this case, we set $w_{n}^{\prime}=1\left(n=1,2, \cdots, W_{T}\right)$ and $w_{n}^{\prime}=0\left(n=W_{T}+1, W_{T}+2, \cdots, N\right)$.

Case 2: There is one or more modified number not smaller than one.

In this case, two main steps are involved.

Step 1: Rank the modified number in descending order and save the corresponding subscripts into set $\left\{G_{1}, \cdots, G_{N}\right\}$. Without losing generality, we notate the modified number as $W_{1}^{\prime \prime \prime} \geq \cdots \geq W_{N}^{\prime \prime \prime}$, where $W_{n}^{\prime \prime \prime} \geq 1(n=1,2, \cdots, r)$ and $W_{n}^{\prime \prime \prime}<1(n=r+1, r+2, \cdots, N)$. Then, we set

$$
W_{n}^{\prime \prime \prime}=1(n=1,2, \cdots, r)
$$

Step 2: Increase the value $W_{n}^{\prime \prime}(n=r+1, r+2, \cdots, N)$ by $\frac{1}{N-r} \sum_{n=1}^{r}\left(W_{n}^{\prime \prime \prime}-1\right)$ to preserve the total number of APs unchanged, and then save $\left\{W_{1}^{\prime \prime \prime}, \cdots, W_{N}^{\prime \prime \prime}\right\}$ into set $\left\{w_{1}^{\prime}, \cdots, w_{N}^{\prime}\right\}$. 


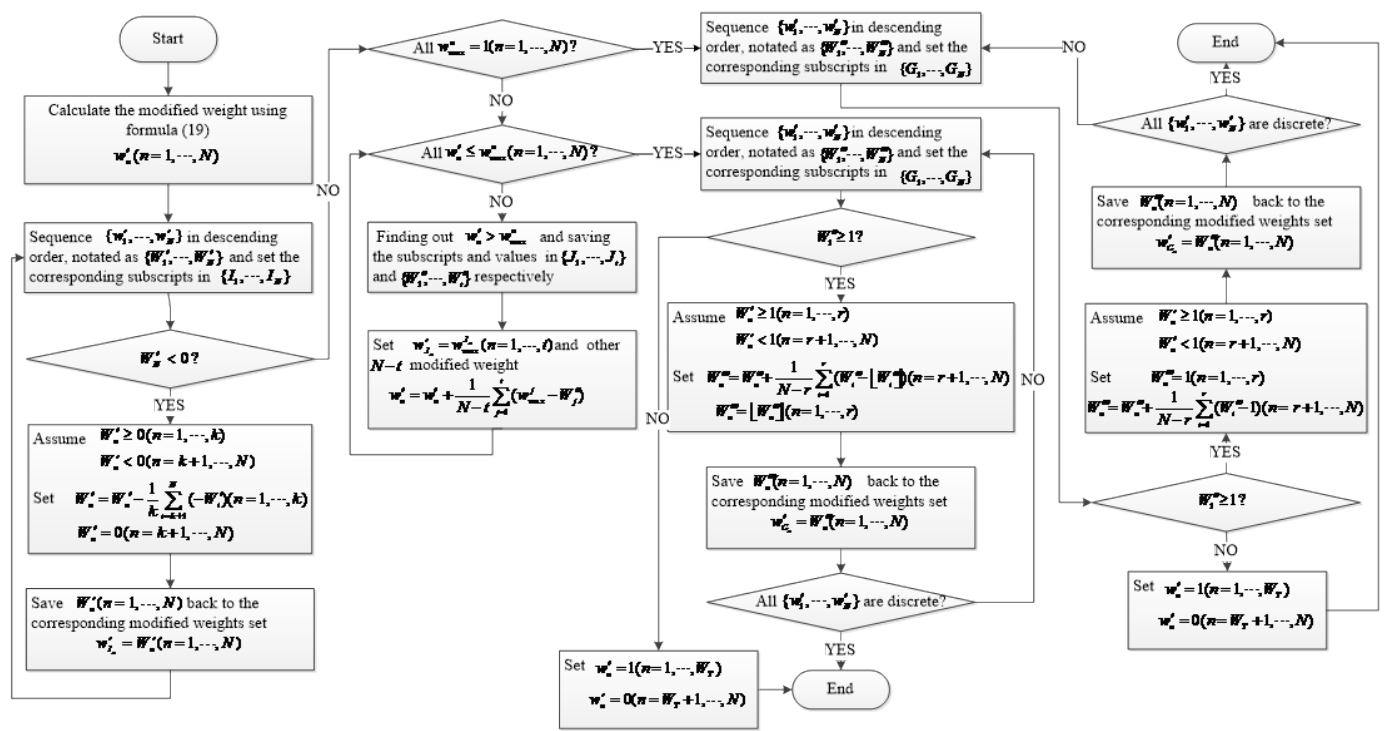

Fig. 3. Flowchart of the proposed FWA-GPC algorithm

\section{Experimental Classification Results and Analysis}

\subsection{Experimental Setup}

To evaluate the performance of the proposed approach, we conduct the experiments on the same floor in a building, where the dimensions are $64.6 \mathrm{~m}$ by $18.5 \mathrm{~m}$, as shown in Fig.4. Due to the space limitation, there are in total seven candidate AP locations, which are labeled as (1), (2), .., (7) respectively. We select a SAMSUNG mobile phone with Samsung Exynos CPU 4412 and 1 Gigabyte memory to record the Wi-Fi RSS data by using our developed software. This software is capable to report the RSS (in $\mathrm{dBm}$ ) and the corresponding MAC addresses of the hearable APs, as shown in Fig. 5. There are in total 363 RPs (with "“") separated with the same interval $0.6 \mathrm{~m}$. At each RP, we record 60 RSS samples under each measuring time (i.e., in the morning, afternoon, and night) and each direction (i.e., to the east, south, west, and north) for the testing. In addition, we evaluate the proposed approach by the comparison with the conventional Random, Max Mean, and InfoGain approaches in the aspects of positioning error and power consumption.

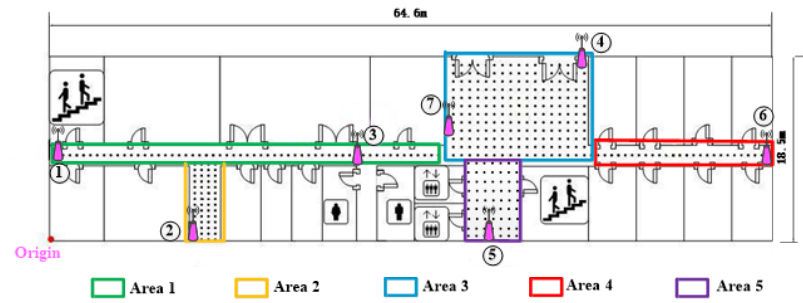

Fig. 4. Environmental layout

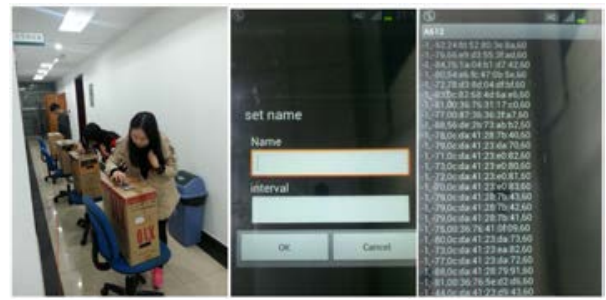

Fig. 5. Some photos about the data recording and our software

\subsection{Discussion of value $d$}

To investigate the impact of value $\boldsymbol{d}$ in objective function on AP optimization and positioning accuracy, we adopt four configurations in the experiment, where the value $d$ equals $1 \mathrm{~m}, 3 \mathrm{~m}$, 
$5 \mathrm{~m}$, and $7 \mathrm{~m}$ respectively. The value $\beta$ is set as 2 and spare APs are not considered (i.e. $\left.w_{\max }^{n}=1\right)$. Table 2 illustrates the optimal group of candidate AP locations under different AP number as the value $d$ varies from $1 \mathrm{~m}$ to $7 \mathrm{~m}$. Obviously, different value $d$ results in different optimal groups of candidate AP locations.

Table 2. Optimal groups of candidate AP locations under different value $d$

\begin{tabular}{|c|c|c|c|c|}
\hline \multirow[t]{2}{*}{ Number of APs } & \multicolumn{4}{|c|}{ Optimal candidate AP location IDs } \\
\hline & $d=1 \mathrm{~m}$ & $d=3 \mathrm{~m}$ & $d=5 \mathrm{~m}$ & $d=7 \mathrm{~m}$ \\
\hline 1 & (1) & (1) & (5) & (5) \\
\hline 2 & (1)(3) & (1)(6) & (5)(7) & (5)(7) \\
\hline 3 & (1)(2)(3) & (1)(3) 6 & (3)(5)(7) & (4) (5) 7 \\
\hline 4 & (1)(2)(3) (7) & (1)(2)(3)(6) & (3)(5)(6) 7 & (3) (4) (5) 7 ) \\
\hline 5 & (1)(2)(3)(7) & (1)(2)(3)(5) & (1)(3)(5)(6) & (3)(4) (5) 6 (7) \\
\hline 6 & (1)(2)(3)(4)(6)(7) & (1)(2)(3)(5)6(7) & (1)(3)(4)(5)(6)(7) & (2)(3)(4)(5)(7) \\
\hline
\end{tabular}

Fig. 6. shows the impact of value $d$ on positioning accuracy under different number of APs. It illustrates the Cumulative Distribution Functions (CDFs) of errors when the optimal groups of candidate AP locations are selected.

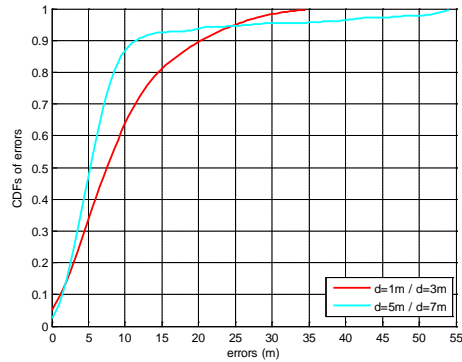

(a) 1 APs

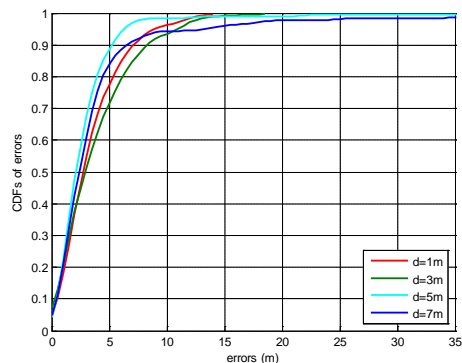

(d) 4 APs

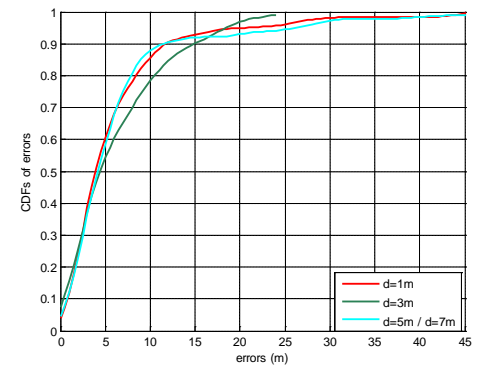

(b) 2 APs

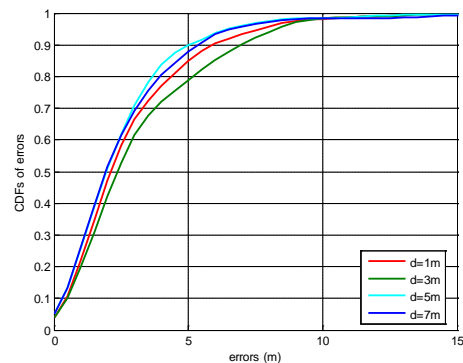

(e) 5 APs

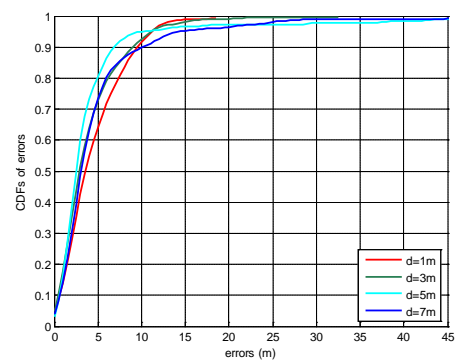

(c) 3 APs

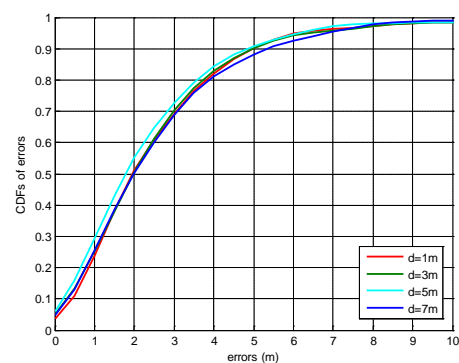

(f) 6 APs

Fig. 6. CDFs of errors under different value $d$

To be clearer, in Fig. 7, the mean error versus number of APs under different value $d$ is given. 


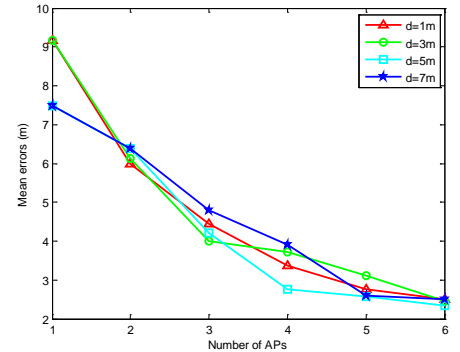

Fig. 7. Mean error versus number of APs under different value $d$

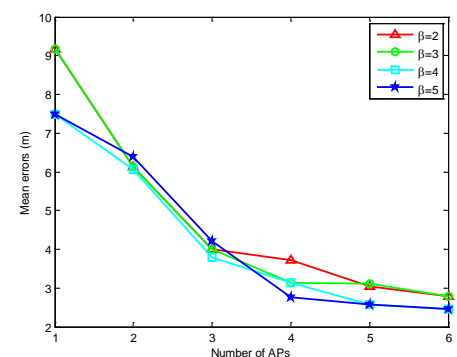

Fig. 9. Mean errors versus AP number under different value $\beta$

From Fig. 6 and Fig. 7, we observe that i) the positioning accuracy gradually improves as the number of APs increases; ii) the steepness of the curve of mean errors generally decreases with the increase of AP number; and iii) the value $d$ has significant impact on the AP optimization for indoor positioning especially under the small number of APs.

\subsection{Discussion of value $\beta$}

To investigate how the value $\beta$ in objective function affects the AP optimization, we vary the value $\beta$ from 2 to 5 . The value $d$ is fixed as 3 and spare APs are not considered. Table 3 illustrates the optimal groups of candidate AP locations under different AP number.

Table 3. Optimal groups of candidate AP locations under different value $\beta$

\begin{tabular}{|c|c|c|c|c|}
\hline \multirow[t]{2}{*}{ Number of APs } & \multicolumn{4}{|c|}{ Optimal candidate AP location IDs } \\
\hline & $\beta=2$ & $\beta=3$ & $\beta=4$ & $\beta=5$ \\
\hline 1 & (1) & (1) & (5) & (5) \\
\hline 2 & (1)(6) & (1)(6) & (3)(5) & (3)(5) \\
\hline 3 & (1)(3)(6) & (1)(3)(6) & (1)(3)(5) & (3)(5)(7) \\
\hline 4 & (1)(2)(3) & (1)(3)(5) & (1)(3)(5) & (3)(5)(6)(7) \\
\hline 5 & (1)(2)(3)(5)(6) & (1)(3)(5)(6)(7) & (1)(3)(5)(6)(7) & (1)(3)(5)(6)(7) \\
\hline 6 & (1)(2)(3)(5)(6)(7) & (1)(2)(3)(5)(6)(7) & (1)(3)(4)(5)(6)(7) & $\begin{array}{c}\text { (1) (4)(5)(6) } \\
\text { (7) }\end{array}$ \\
\hline
\end{tabular}

Likewise, the optimal groups of candidate AP locations are different with respect to different value $\beta$ under the same AP number. Fig. 8 and Fig. 9 show the CDFs of errors and the mean error versus number of APs under different value $\beta$ respectively.

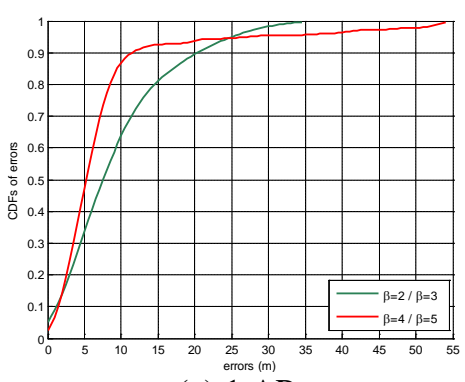

(a) $1 \mathrm{AP}$

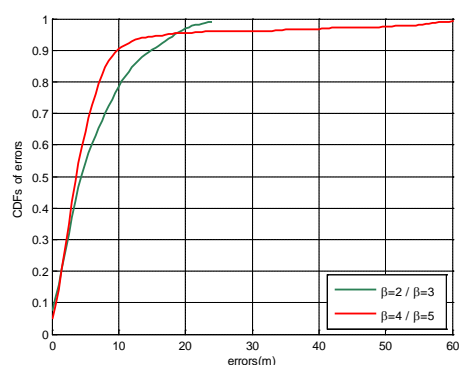

(b) 2 APs

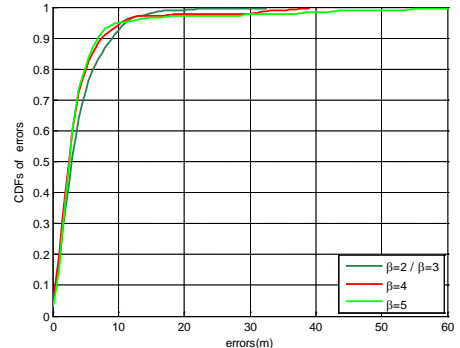

(c) 3 APs 


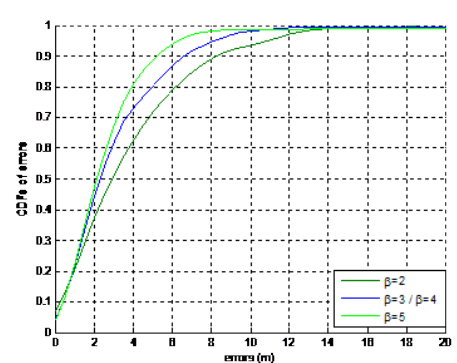

(d) 4 APs

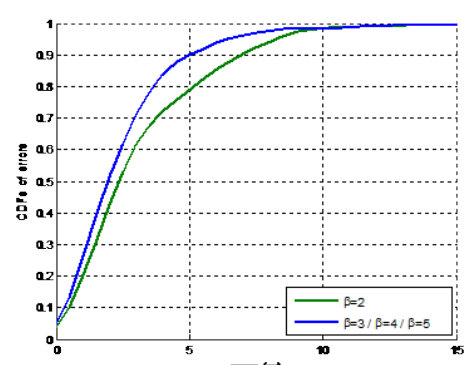

(e) 5 APs

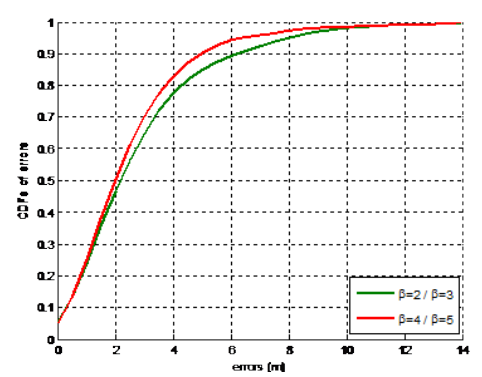

(f) 6 APs

Fig. 8. CDFs of errors under different value $\boldsymbol{\beta}$

The experimental results indicate that the value $\beta$ has significant impact on AP optimization for indoor positioning especially under the small number of APs.

\subsection{Discussion of value $w_{\max }^{n}$}

The experiments conducted above do not consider the spare APs, that is to say, there is only one AP at every candidate AP location. But in some circumstances, it is indispensable to consider the spare APs. For instance, the candidate AP locations AP(4) and AP(7) in area 3 can be deployed with the spare APs to guarantee the quality of communication, but the key problem is to choose the proper candidate AP locations to be deployed with the spare APs from the viewpoint of positioning. In our system, we solve this problem effectively by setting an upper-bound parameter $\boldsymbol{w}_{\max }^{n}$.

In our environment, there are two areas facing the alternative candidate AP locations, Area 1 and 3. For simplicity, we focus on these two areas with only one spare AP. Then, two cases are discussed respectively as follows.

Case 1: Spare AP is in Area 1.

In this case, we set $\boldsymbol{w}_{\max }^{1}=2$ and $\boldsymbol{w}_{\max }^{3}=2$. Fig. 10 shows the two possible results of AP optimization by the proposed FWA-GPC algorithm with the spare AP located in Area 1.

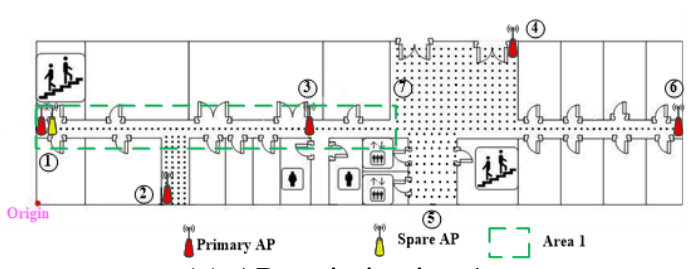

(a) AP optimization 1

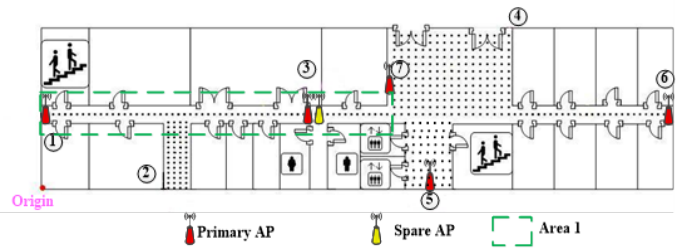

(b) AP optimization 2

Fig. 10. Two possible results of AP optimization with one spare AP in Area 1

Fig. 11 and Fig. 12 compare the positioning accuracy between two circumstances, in which the primary APs at (1) and (3) are invalid respectively with no spare AP as a supplement in Area 1. Fig. 11 shows that the probability of errors within $4 \mathrm{~m}$ is about $68 \%$ when the AP at (1) is invalid, which is about 6 percentages lower than the one when the AP at (3) is invalid. That is to say, the AP at (1) is more beneficial in positioning compared with the one at (3) under AP optimization 1. Likewise, as can be seen from Fig. 12, the AP at (3) performs better than the one at (1) in terms of positioning accuracy under AP optimization 2. Consequently, we can conclude that the proposed FWA-GPC algorithm is capable of choosing the most proper locations for the spare APs from the viewpoint of positioning. 


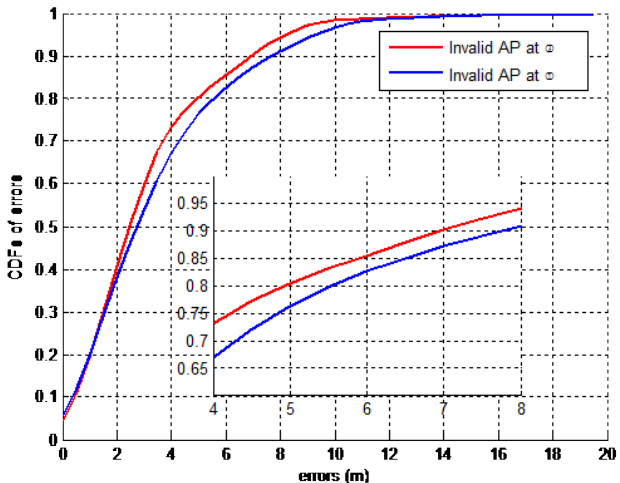

Fig. 11. CDFs of errors under AP optimization 1 with no spare AP in Area 1

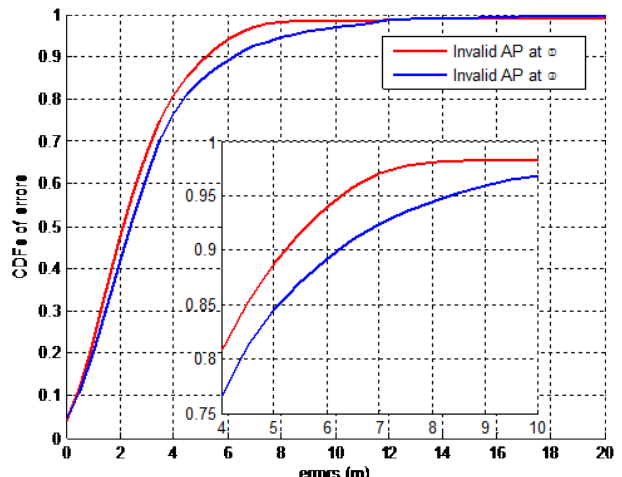

Fig. 12. CDFs of errors under AP optimization 2 with no spare AP in Area 1

Case 2: Spare AP is in Area 3.

In this case, we set $\boldsymbol{w}_{\max }^{4}=2$ and $\boldsymbol{w}_{\max }^{7}=2$. Fig. 13 shows the two possible results of AP optimization by the proposed FWA-GPC algorithm with the spare AP located in Area 3.

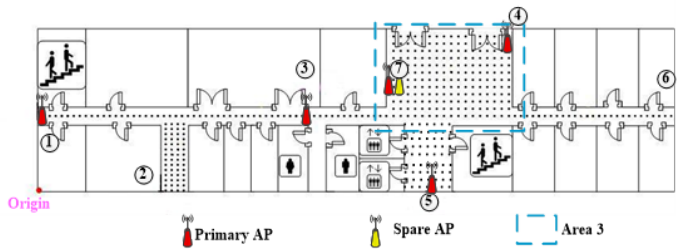

(a) AP optimization 1

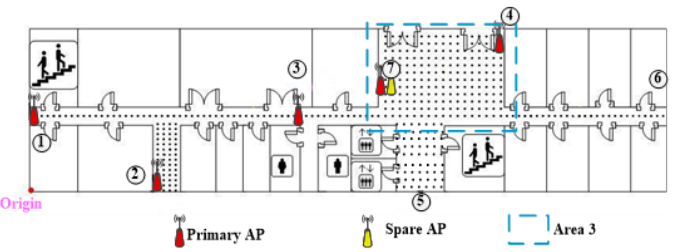

(b) AP optimization 2

Fig. 13. Two possible results of AP optimization with the spare AP in Area 3

Fig. 14 and Fig. 15 compare the positioning accuracy between two circumstances, in which the APs at (4) and (7) are invalid respectively with no spare AP as a supplement Area 3. These results further demonstrate that our approach is promising with the spare APs optimization.

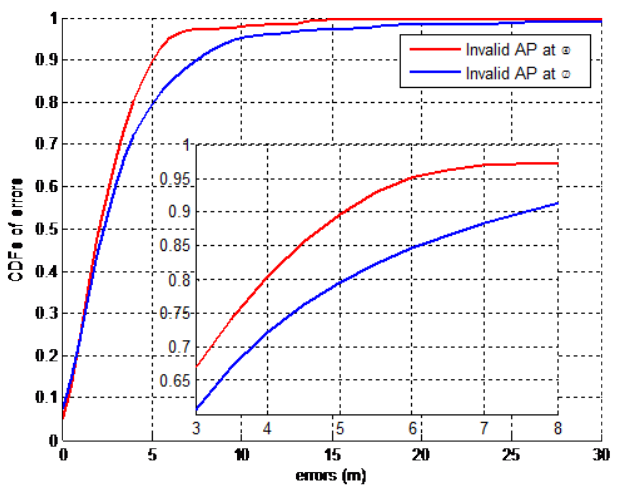

Fig. 14. CDFs of errors under AP optimization 1 with no spare AP in Area 3

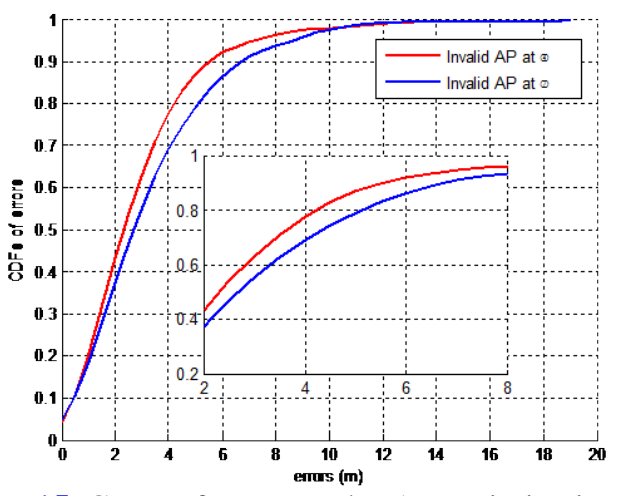

Fig. 15. CDFs of errors under AP optimization 2 with no spare AP in Area 3

The above experiments are about two quintessential examples to demonstrate that our approach is effective for the spare APs optimization in Area 1 and 3. As a matter of fact, our approach is not limited to these two areas. Gratifyingly, we can deal with it similarly to set an 
upper-bound parameter $w_{\max }^{n}$. Particularly, when all the candidate AP locations in the environment are not restricted to only one AP, which means $w_{\max }^{n}=2(n=1,2, \cdots, 7)$, the total power $W_{T}$ (i.e. total number of APs) will become pretty important to the AP optimization for indoor positioning. Fig. 16 shows the number of APs deployed at each candidate AP location as the total power increases from 6 to 8 . From this figure, we can find that when the total power is 6, the number of APs deployed at each candidate AP location is not larger than one, while when the total power increases to 7 or larger, some candidate AP locations will be deployed with more than one AP.

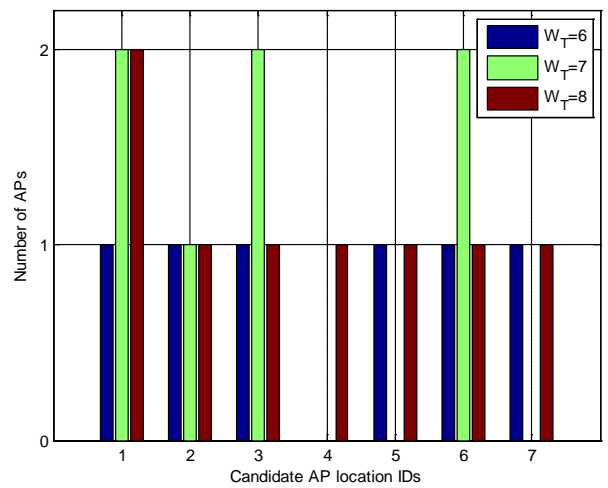

Fig. 16. AP optimization under different total power

\subsection{Algorithms Comparison}

In this section, we will continue to compare the CDFs of errors among different AP optimization algorithms mentioned in the previous section 1 . Fig. 17 shows the CDFs of errors obtained by the conventional InfoGain, MaxMean, Random, and proposed FWA-GPC algorithms.

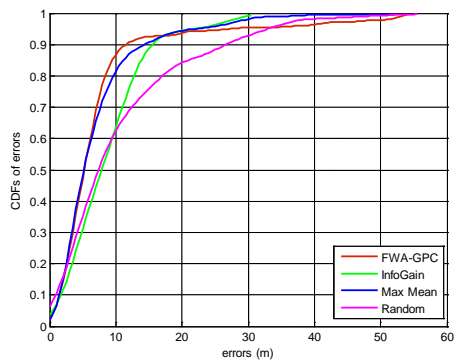

(a) $1 \mathrm{AP}$

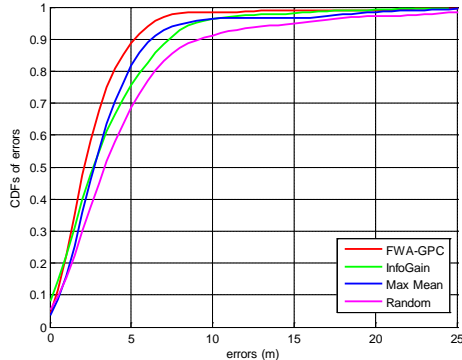

(d) 4 APs

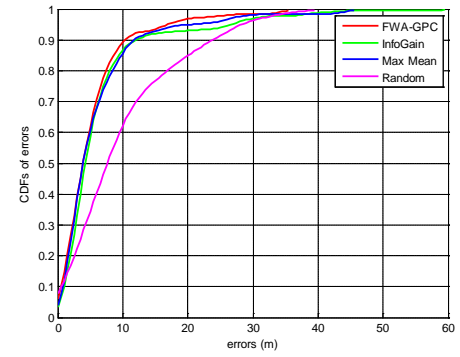

(b) 2 APs

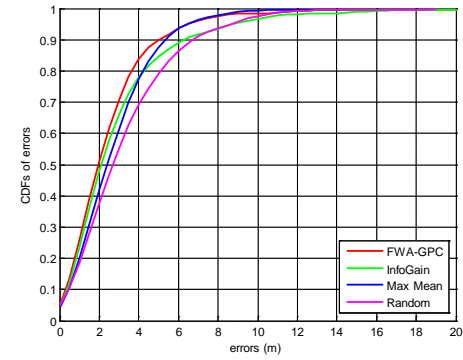

(e) 5 APs

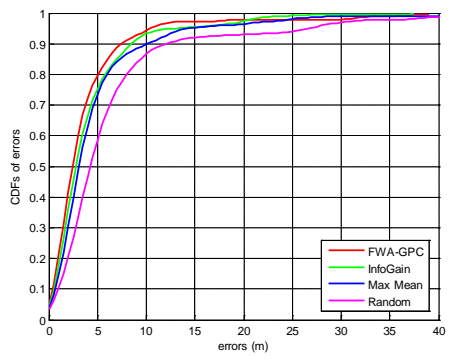

(c) 3 APs

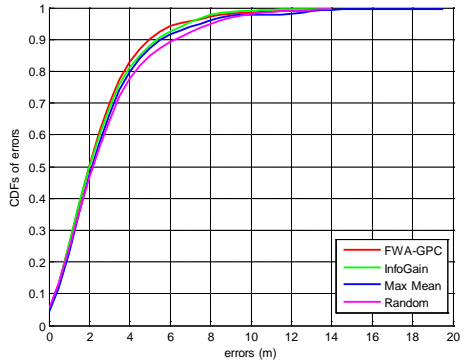

(f) 6 APs

Fig. 17. CDFs of errors by different AP optimization algorithms 
From this figure, it is demonstrated that the proposed FWA-GPC algorithm outperforms the other four in terms of positioning accuracy. As an example, probabilities of errors within $10 \mathrm{~m}$ by the InfoGain, Max Mean, and Random are $62.50 \%, 81.00 \%$, and $61 \%$ respectively, which are smaller than the one by the FWA-GPC, $88.00 \%$. The probabilities of errors within $5 \mathrm{~m}$ by the InfoGain, Max Mean, and Random are about 14, 8.5, and 21.5 percentages respectively lower than the one by the FWA-GPC.

Furthermore, by considering the 4 APs scenario, Table 4 shows some numerical results about the positioning accuracy in terms of mean of errors, Standard Deviation (STD) of errors, median of errors, 50\% Circular Error Probable (CEP), 67\% CEP, and 90\% CEP. The CEP is defined as the radius of the circle that has its center at the true location and contains the location estimates with a probability. Table 4 shows a similar tendency that the proposed FWA-GPC algorithm provides better positioning accuracy compared with the traditional ones. Table 4. Numerical results about the positioning accuracy by different AP optimization algorithms with four APs

\begin{tabular}{|l|c|c|c|c|c|c|}
\hline Algorithms & Mean & STD & Median & 50\%CEP & 67\%CEP & 90\%CEP \\
\hline FWA-GPC & 2.77 & 3.74 & 2.00 & 2.30 & 3.10 & 5.10 \\
\hline InfoGain & 3.46 & 3.43 & 2.6 & 2.70 & 4.00 & 7.40 \\
\hline Max Mean & 3.58 & 4.17 & 2.68 & 2.75 & 3.80 & 6.70 \\
\hline Random & 4.13 & 5.15 & 3.34 & 3.50 & 4.00 & 8.50 \\
\hline
\end{tabular}

In Fig. 18, Fig. 19, and Fig. 20, the mean of errors, median of errors, and STD of errors versus number of APs are illustrated respectively. These results strongly support that the positioning accuracy generally improves as the number of APs increases. In addition, they further demonstrate that the proposed FWA-GPC algorithm is capable to achieve the overall best positioning performance. To verify the advantage of the FWA-GPC in power consumption, Fig. 21 compares the power consumed by the FWA-GPC, CWA-GPC mentioned in section 2, InfoGain, and Max Mean algorithms.

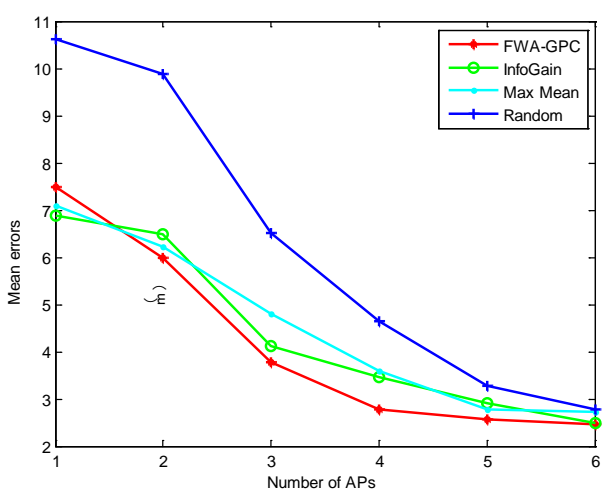

Fig. 18. Mean of errors versus number of APs

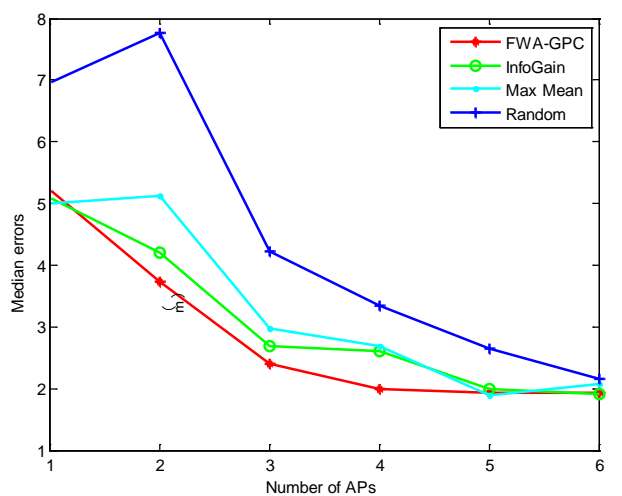

Fig. 19. Median of errors versus number of APs 


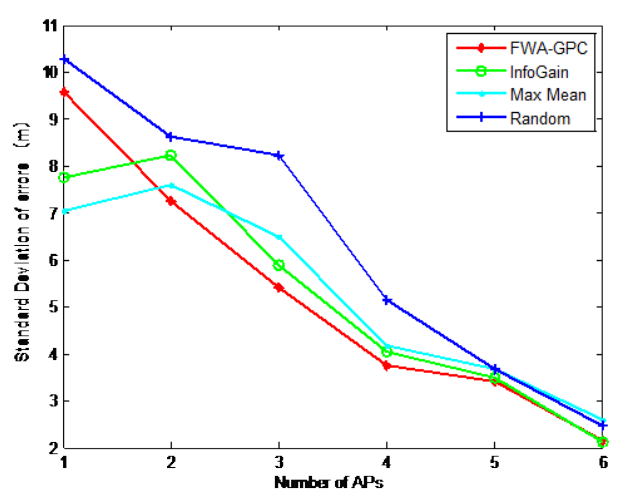

Fig. 20. STD of errors versus number of APs

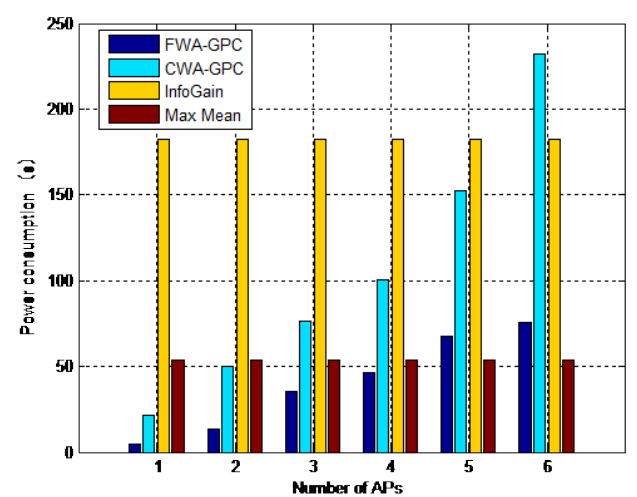

Fig. 21. Power consumption by different AP optimization algorithms

From Fig. 21, we can find that the power consumed by the CWA-GPC increases steeply as the number of APs increases since the CWA-GPC needs multiple iterations to find the optimal value $\boldsymbol{\mu}$. In contrast, the power consumption by the FWA-GPC increases slowly, which benefits from the dodge of searching for the optimal value $\mu$. In addition, the power consumed by the InfoGain and Max Mean remains unchanged with the increase of AP number due to the reason that these two algorithms require traversing all the candidate AP locations regardless of AP number.

\subsection{Testing Results in another Environment}

To demonstrate the scalability of the proposed approach, we repeat the same experimental procedure in another environment with the dimensions of $57 \mathrm{~m}$ by $25 \mathrm{~m}$ on the same floor in a building. In this environment, the number of candidate AP locations is 9, as shown in Fig. 22. Due to the space limitation, in this section, we only investigate the positioning accuracy and power consumption by different AP optimization algorithms including the InfoGain, Max Mean, Random, and proposed FWA-GPC algorithms.
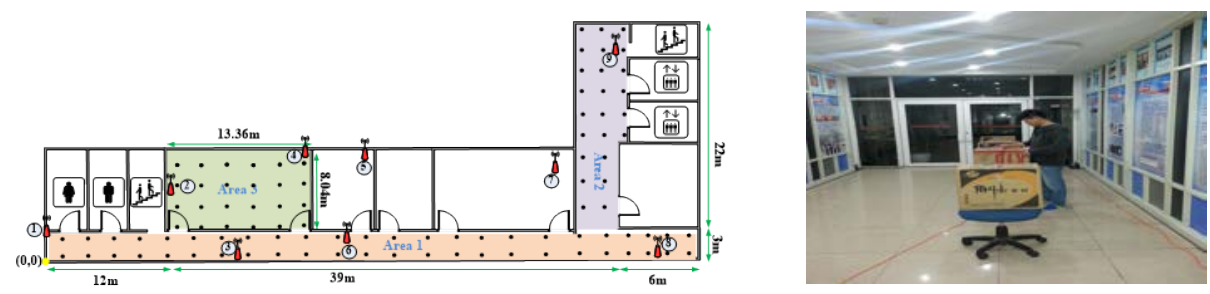

Fig. 22. Layout of another environment

Similarly, we show the mean of errors, median of errors, and STD of errors versus number of APs in Fig. 23, Fig. 24, and Fig. 25 respectively. Fig. 26 compares the power consumption by the CWA-GPC, InfoGain, Max Mean, and proposed FWA-GPC algorithms. 


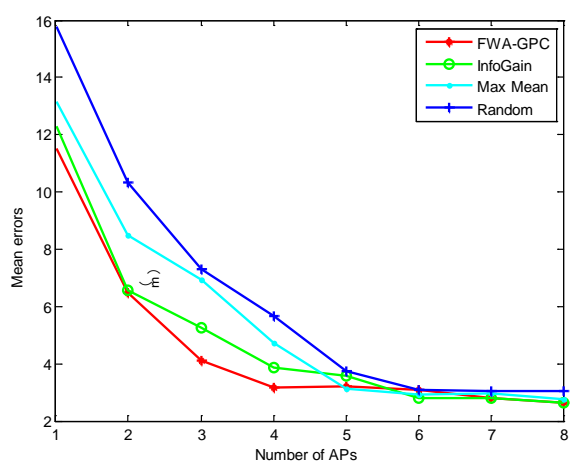

Fig. 23. Mean of errors versus number of APs

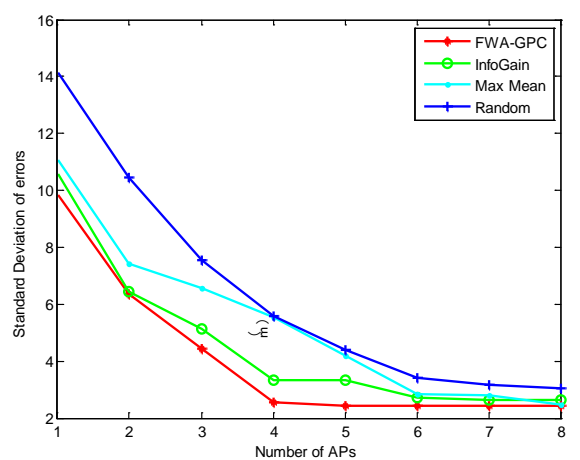

Fig. 25. STD of errors versus number of APs

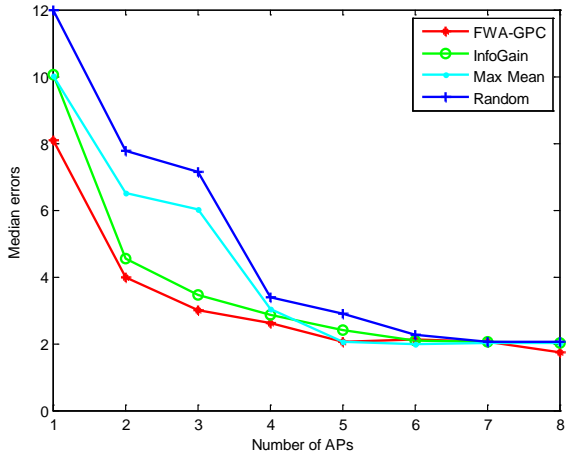

Fig. 24. Median of errors versus number of APs

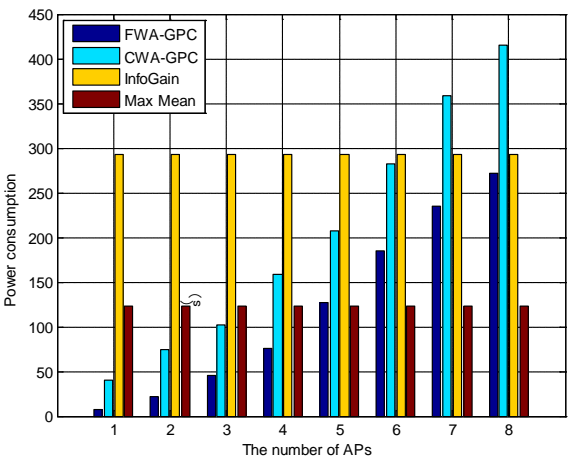

Fig. 26. Power consumption by different AP optimization algorithms

Obviously, the above experimental results obtained in another environment demonstrate that the proposed approach featured with well scalability compared with the conventional ones in terms of localization accuracy and power consumption.

\section{Conclusion}

The proposed AP optimization approach, FWA-GPC, can be used to recommend the best group of candidate AP locations which most contributes to the location fingerprint discrimination and power consumption for indoor positioning. Although the huge power consumption problem definitely exists when there are a large number of APs in target environment, the conventional AP optimization approaches treat this problem independent from the design of indoor positioning systems. Unlike the conventional ones, the proposed FWA-GPC focuses on measuring the location fingerprint discrimination of the APs with a given total power, rather than assuming the equal power consumed by each AP. The proposed approach examined in two representative indoor environments covered by multiple Wi-Fi APs. The experimental results report that the proposed FWA-GPC reduces the average positioning error by $24.91 \%-29.24 \%$ and power consumption by $15.98 \%-30.03 \%$, compared with the conventional Random, Max Mean, and InfoGain approaches. Furthermore, exploring the AP optimization approach for multi-floor indoor Wi-Fi environment forms an interesting topic in future. 


\section{References}

[1] M. Chon and H. Cha, "Life Map: A smartphone-based context provider for location-based services, ” IEEE Pervasive Computing, vol. 10, no. 2, pp. 58-67, 2011. Article (CrossRef Link)

[2] Z. Sun, W. Zhang, and Y. Xie, "Analysis of environmental factors for a WLAN location system using an estimated radio map," in Proc. of IEEE International Conference on Communication Technology, pp. 946-949, 2010. Article (CrossRef Link)

[3] S. Tang, B. Tok, and M. Hannehan, "Passive Indoor Positioning System (PIPS) Using Near Field Communication (NFC) Technology," in Proc. of International Conference on Developments of E-Systems Engineering, pp. 150-155, 2015. Article (CrossRef Link)

[4] Y. Tian, W. Wang, C. Ahn, T. Omori, and K. Hashimoto, "The accuracy improvement using WLAN for indoor positioning system,” in Proc. of International Symposium on Intelligent Signal Processing and Communication Systems, pp. 474-478, 2015. Article (CrossRef Link)

[5] S. Santosh, G. Kwon, S. Shin, S. Hwang, and J. Pyun, "Beacon based indoor positioning system using weighted centroid localization approach," in Proc. of International Conference on Ubiquitous and Future Networks, pp. 1016-1019, 2016. Article (CrossRef Link)

[6] N. Milos and M. Aleksandar, "Comparative analysis of RSSI, SNR and Noise level parameters applicability for WLAN positioning purposes,” IEEE EUROCON, pp. 1892-1900, 2009.

Article (CrossRef Link)

[7] X. Fu, N. Zhang, A. Pingley, W. Yu, J. Wang, and W. Zhao, “The Digital Marauder's Map: A Wi-Fi Forensic Positioning Tool,” IEEE Transactions on Mobile Computing, vol. 11, no. 3, pp. 377-389, 2012. Article (CrossRef Link)

[8] A. Irish, J. Isaacs, and F. Qui, "Belief propagation based localization and mapping using sparsely sampled GNSS SNR measurements,” in Proc. of IEEE International Conference on Robotics and Automation, pp. 1977-1982, 2014. Article (CrossRef Link)

[9] R. Kaune, “Accuracy studies for TDOA and TOA localization," in Proc. of International Conference on Information Fusion, pp. 408-415, 2012. Article (CrossRef Link)

[10] K. Mohamed, "Time of arrival (TOA)-based direct location method," in Proc.of International Radar Symposium, pp. 812-815, 2015. Article (CrossRef Link)

[11] S. Kariman, M. Aziz, and T. Matsumoto, "A hybrid TOA and RSS-based factor graph for wireless geolocation technique,” in Proc. of International Colloquium on Signal Processing \& Its Applications, pp. 140-145, 2016. Article (CrossRef Link)

[12] S. Tomic, M. Beko, and R. Dinis, "Distributed RSS-AoA Based Localization With Unknown Transmit Powers,” IEEE Wireless Communications Letters, vol. 5, no. 4, pp. 392-395, 2016. Article (CrossRef Link)

[13] K. Chintalapudi, A. Padmanabha, and V. Padmanabhan, "Indoor Localization without the Pain," MobiCom, pp. 173-184, 2010. Article (CrossRef Link)

[14] S. P. Kuo and Y. C. Tseng, "Discriminant minimization search for large-scale RF-based localization systems,” IEEE Transaction on Mobile Computing, vol. 10, no. 2, pp. 291-304, 2011. Article (CrossRef Link)

[15] H. Wang, X. Zhang, Y. Gu, L. Zhang, and J. Li, "Indoor Wi-Fi RSS-fingerprint location algorithm based on sample points clustering and AP reduction," in Proc. of International Conference on Intelligent Control and Information Processing, pp. 264-267, 2015. Article (CrossRef Link)

[16] A. Abusara, M. Hassan, and H. Ismail, "RSS fingerprints dimensionality reduction in WLAN-based indoor positioning," Wireless Telecommunications Symposium, pp. 1-6, 2016. Article (CrossRef Link)

[17] S. Fang, T. Lin, and P. Lin, “Location Fingerprinting in a Decorrelated Space,” IEEE Transactions on Knowledge and Data Engineering, vol. 20, no. 5, pp. 685-691, 2008. Article (CrossRef Link)

[18] H. Lim, L. Kung, J. C. Hou, and H. Luo, “Zero-Configuration, Robust Indoor Localization: Theory and Experimentation,” in Proc. of IEEE INFOCOM, pp. 1-12, 2006. Article (CrossRef Link)

[19] S. Fang and T. Lin, "Principal Component Localization in Indoor WLAN Environments," IEEE Transactions on Mobile Computing, vol. 11, no. 1, pp. 100-110, 2012. Article (CrossRef Link) 
[20] M. Zhou, Q. Pu, K. Xu, X. Huang, and Z. Tian, "Location Fingerprint Discrimination Maximization for Indoor WLAN Access Point Optimization Using Fast Discrete Water-Filling,” in Proc. of IEEE GLOBECOM, pp. 1-6, 2015. Article (CrossRef Link)

[21]D. Sanchez, A. Quintana, and J. Navarro, "WLAN Location Determination Using Probability Distributions with Search Area Reduction via Trilateration,” in Proc. of International Conference on Wireless and Mobile Communications, pp. 328-333, 2009. Article (CrossRef Link)

[22]Z. Li, T. Braun, and C. Dimitrova, "A passive WiFi source localization system based on fine-grained power-based trilateration," in Proc. of International Symposium on a Mobile and Multimedia Networks, pp. 1-9, 2015. Article (CrossRef Link)

[23] S. Eisa, J. Peixoto, F. Meneses, and A. Moreira, "Removing Useless APs and Fingerprints from WiFi Indoor Positioning Radio Maps,” in Proc. of International Conference on Indoor Positioning and Indoor Navigation, pp. 1-7, 2013. Article (CrossRef Link)

[24] S. Fang and T. Lin, "A Novel Access Point Placement Approach for WLAN-based Location Systems,” IEEE WCNC, pp. 1-4, 2010. Article (CrossRef Link)

[25] Y. Zheng, B. Oumaya, and C. Alexandre, "A new approach to design a WLAN-based positioning system,” in Proc. of International Conference on Signal Processing and Communication Systems, pp. 1-8, 2010. Article (CrossRef Link)

[26] T. Jin, X. Qiu, D. Hu, and C. Ding, "Estimation Accuracy and Cramér-Rao Lower Bounds for Errors in Multichannel HRWS SAR Systems," in Proc. of IEEE Geoscience and Remote Sensing Letters, vol. 13, no. 12, pp. 1772-1776, 2016. Article (CrossRef Link)

[27]Z. Deng, Y. Xu, and L. Ma, “Joint access point selection and local discriminant embedding for energy efficient and accurate wi-fi positioning," KSII Transactions on internet and information systems, vol. 6, no. 3, pp. 794-814, 2012. Article (CrossRef Link)

[28] G. Chen, Y. Zhang, F. Luan, L. Xiao, and X. Zhang, "Optimization of AP Placement in Indoor Fingerprint Positioning,” in Proc. of International Conference on ICT Convergence, pp. 98-100, 2013. Article (CrossRef Link)

[29] X. Du and K. Yang, “A Map-Assisted WiFi AP Placement Algorithm Enabling Mobile Device’s Indoor Positioning,” IEEE Systems Journal, vol. PP, no. 99, pp. 1-9, 2016. Article (CrossRef Link)

[30] K. Sui, S. Sun, Y. Azzabi, X. Zhang, and Y. Zhao, "Understanding the Impact of AP Density on WiFi Performance Through Real-World Deployment,” in Proc. of IEEE Conference Publications, pp. 1-6, 2016. Article (CrossRef Link)

[31] L. Ma, X. Zheng, Y. Lu, and X. Tan, "Optimization for the Deployment and Transmitting Power of AP Based on Green WLAN," in Proc. of International Conference on Instrumentation, Measurement, Computer, Communication and Control, pp. 129-134, 2013. Article (CrossRef Link)

[32] B. Phillip and D. Steven, “AP Association Optimization and CCA Threshold Adjustment in Dense WLANs,” in Proc. of IEEE GLOBECOM Workshops, pp. 1-6, 2015. Article (CrossRef Link)

[33] S. Fang, T. Lin, and P. Lin, “Location Fingerprinting in a Decorrelated Space,” IEEE Transactions on Knowledge and Data Engineering, vol. 20, no. 5, pp. 685-691, 2008. Article (CrossRef Link)

[34] M. Youssef, A. Agrawala, and A. Udaya Shankar, "WLAN Location Determination via Clustering and Probability Distributions," in Proc. of IEEE International Conference on Pervasive Computing and Communications, pp. 143-150, 2003. Article (CrossRef Link)

[35] Y. Chen, Q. Yang, J. Yin, and X. Chai, "Power-Efficient Access-Point Selection for Indoor Location Estimation,” IEEE Transactions on Knowledge and Data Engineering, vol. 18, no. 7, pp. 877-888, 2006. Article (CrossRef Link)

[36] Y. Shi, D. Guo, L. Lu, and X. Duan, “A modified water-filling algorithm of power allocation,” in Proc. of IEEE Conference on Information Technology, Networking, Electronic and Automation Control, pp. 1125-1129, 2016. Article (CrossRef Link)

[37] K. Zhou and L. Cai, “A Dynamic Water-Filling Method for Real-Time HVAC Load Control Based on Model Predictive Control,” IEEE Transactions on Power Systems, vol. 30, no. 3, pp. 1405-1414, 2015. Article (CrossRef Link) 
[38] L. Zhao, Y. Wang, and P. Charge, "Efficient iterative water-filling power allocation method in MU-MIMO broadcast channels," in Proc. of Military Communications and Information Systems Conference, pp. 1-5, 2013. Article (CrossRef Link)

[39] H. Wei and P. Sun, "Lagrange multiplier based domain decomposition method with non-matching grids for time-dependent equations," in Proc. of International Conference on High Performance Computing in the Asia-Pacific Region, pp. 998-1000, 2000. Article (CrossRef Link)

[40] X. Ling, B. Wu, P. Ho, F. Luo, and L. Pan, "Fast Water-Filling for Agile Power Allocation in Multi-Channel Wireless Communications,” IEEE Communications Letters, vol. 16, no. 8, pp. 1212-1215, 2012. Article (CrossRef Link)
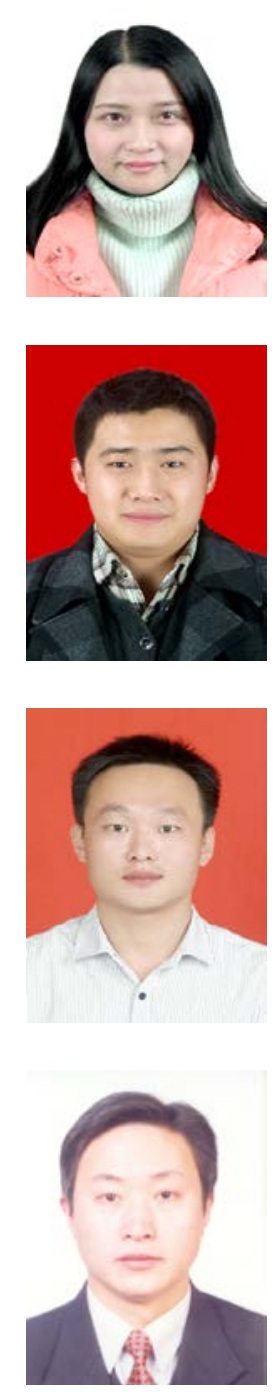

Qiaolin Pu received her Bachelor degree and Master degree from Chongqing University of Posts and Telecommunications (CQUPT). She is currently working in the Chongqing Key Lab of Mobile Communications Technology, CQUPT. Her research focuses on wireless localization, networking optimization, statistical theories to communication, and resource allocation problems.

Mu Zhou received the Bachelor degree, Master degree, and PHD degree from Harbin Institute of Technology. He is now a professor in CQUPT, his research focuses on wireless localization, machine learning, artificial intelligence, and convex optimization.

Fawen Zhang received the Bachelor degree from Luoyang normal college, received the Master degree degree from Guangzhou University. He is now working in Computer Science \& Technology College, CQUPT.

Zengshan Tian received the Bachelor degree from Zhengzhou University, Received the Master degree and PHD degree from University of Electronic Science and Technology of China. He is now a professor in CQUPT, his research focuses on localization in cellular network, personal communication, precise localization and attitude measurement with GPS, data compression, and data fusion. 